\title{
Fracture Process Characterization of Fiber-Reinforced Dental Composites Evaluated by Optical Coherence Tomography, SEM and Optical Microscopy
}

\author{
Tibério C. U. MATHEUS ${ }^{1}$ \\ Cynthia M. F. KAUFFMAN ${ }^{2}$ \\ Ana K. S. BRAZ 3 \\ Cláudia C. B. O. MOTA ${ }^{3}$ \\ Anderson S. L. GOMES 1,3 \\ ${ }^{1}$ Department of Physics, Federal University of Pernambuco, Recife, PE, Brazil \\ ${ }^{2}$ Materials Science Program, Federal University of Pernambuco, Recife, PE, Brazil \\ ${ }^{3}$ Graduate Program in Dentistry, Federal University of Pernambuco, Recife, PE, Brazil
}

\begin{abstract}
This study used optical coherence tomography (OCT) compared to scanning electron microscopy (SEM) and optical microscopy $(\mathrm{OM})$ to evaluate qualitatively crack propagation and final fracture in restorative composite materials - Filtek Z250 and Filtek Z350 (3M ESPE) - with fiber reinforcement after cyclic loading. Samples were made using a split mold. Initially, 3-point bending tests were performed to determine the maximum force and tension at the fracture moment using samples without fiber reinforcement. Then, mechanical cycling tests were performed using samples with glass fiber embedded internally. The failures were analyzed using the 3 methods described before. OCT permitted good characterization of internal crack propagation of the dental composites, which, however, could not be visualized by either SEM or OM. OCT was proven to be laboratory research tool that is easy to use, does not require any specific preparation of the samples, and is less expensive than SEM.
\end{abstract}

Key Words: optical coherence tomography, dental fiber-reinforcement composites, three points bending, scanning electron microscopy, optical microscopy.

\section{INTRODUCTION}

Composites have revolutionized the practice of restorative dentistry. Nowadays, these systems largely dominate the market of cosmetic and restorative materials, and over the past few years have their physical-chemical properties have improved considerably. These resins are classified as composite materials, which are defined as composed of two or three materials with intermediary or superior properties in relation to individual parts. The main elements of modern composites are resin matrix and inorganic particle fillers (1).

Fatigue fracture has been commonly considered the cause of failure in clinical practice of the restorative dentistry. The longevity of restorations depends on several factors including operator's skills, materials and techniques used, criteria for replacement of restorations, patient's oral hygiene, oral health conditions, and their contribution to caries susceptibility of the patient and possibly the means by which the treatment is based. The fatigue on dental restorations of the composite resins is also influenced by water absorption by resin matrix (2) and occlusion cyclical forces (3).

Fiber-reinforcement has been introduced to increase both flexural strength and modulus of these materials (4). However, the inclusion of another reinforcement component can make the material brittle during load. The insertion of a reinforcement component can act as a stress concentrator due to fiber-composite interface. Other stress concentrator points can exist, as

Correspondence: Dr. Tibério C. U. Matheus, Departamento de Física, Universidade Federal de Pernambuco, Avenida Prof. Luiz Freire, S/N, Cidade Universitária, 50670-901 Recife, PE, Brasil. Tel: +55-81-9967-3660. Fax:+55-81-3271-0359. e-mail: tcmatheus@yahoo.com.br 
defects on materials surface and the bigger inorganic particles inside material.

The fracture analysis, named fractography, is well established in the field of biomaterials research, and has been recognized as a powerful analytical tool in dentistry (5). The main advantage of performing an analysis by scanning electron microscopy (SEM) is the excellent resolution obtained, allowing detailed observation of the fracture process. However, this technique promotes only a surface scanning, uses an expensive equipment, and requires specific preparation of the samples, which can generate artifacts and impair the analysis.

Optical coherence tomography (OCT) is a recent imaging technique that uses the properties of light and its interaction with biological tissues to imaging generation of human body parts. OCT is a non-invasive and nondestructive technique that provides images sectioned in real time, high spatial resolution, obtaining images at the microscopic level, considered by many authors as "a biopsy in vivo" (6).

Although studies for this technique in dentistry have been reported earlier, recent results show the potential of this tool for dental materials, particularly, fracture propagation studies in resin composites (7), since it is an in-depth reading. The most successful application of OCT as optical diagnosis is in ophthalmology, where it can be used to create cross-sectional images of retinal pathology with higher resolution than any other noninvasive imaging technique, becoming the standard noncontact noninvasive ophthalmic imaging modalities for routine clinic diagnosis (8). Because of OCT potential to provide morphological information of biological tissues, it has been applied at other medical areas, such early diagnosis of ovarian neoplasia (9) and dermatology (10). Hard tissues like tooth were analyzed by OCT to verify the quality of dental restoration of enamel (11). This study used OCT, SEM and optical microscopy $(\mathrm{OM})$ to evaluate qualitatively the crack propagation in restorative composite materials with fiber reinforcement after cyclic loading.

\section{MATERIAL AND METHODS}

In this study, an optical coherence tomograph (mounted in the Laboratory of Photonics of the Department of Physics, UFPE), a scanning electron microscope (Quanta 200 FEG; Quanta, Hillsboro, OR, USA) and an optical microscope (Olympus-BX 51; Tokyo, Japan) were used. The restorative composite materials used were: Filtek Z250 (3M ESPE, Sumaré SP, Brazil) and Filtek Z350 (3M ESPE). The shade of restorative resins was $\mathrm{A}_{2}$.

\section{Preparation of Samples}

To prepare the samples, a split mold was used with dimensions of $2 \mathrm{~mm} \times 3 \mathrm{~mm} \times 25 \mathrm{~mm}$. Samples were prepared with and without fiber-reinforcement.

For the samples without fiber-reinforcement, resin was inserted into the split mold and covered with a polyester strip. The polyester strip was slightly pressed to allow the extrusion of excess material. Then, the resin was cured with a light-emitting diode (LED) source with power density of $1200 \mathrm{~mW} / \mathrm{cm}^{2}$ and wavelength from 440 to $480 \mathrm{~nm}$ (LED Radii-cal, SDI, Australia). Polymerization was carried out in 3 points of the samples: on its ends and center, and at the top and bottom of the split mold, during $20 \mathrm{~s}$ at each point. After removal of the specimen from the split mold, material excess from the edges was removed with the aid of a scalpel blade.

During the preparation of the fiber-reinforced samples, a glass fiber pre-impregnated with composite resin (Interlig reinforcement fiber; Angelus, Londrina, PR, Brazil) was inserted into the sample to both restorative composite materials described. Therefore, the filling of the split mold with an initial layer of composite resin was followed by the placement of glass fiber preimpregnated with composite resin. Subsequently, a new layer of composite resin was inserted leaving the height of the reinforced specimen with fiber by $2 \mathrm{~mm}$.

The specimens were packed storage in the dark bottle containing distilled water and were incubated at $37^{\circ} \mathrm{C}\left( \pm 2^{\circ} \mathrm{C}\right)$.

\section{Mechanical Tests}

Three-point bending tests were carried out before mechanical cycling tests in samples with fiber reinforcement with the objective of finding out some parameters, as maximum force (in $\mathrm{N}$ ) and maximum tension (in $\mathrm{MPa}$ ) of the fracture of each composite. Five samples without fiber reinforcement of each dental composite were submitted to 3-point bending tests in a universal testing machine (EMIC DL-10000; EMIC, São José dos Pinhais, PR, Brazil) with a load cell capacity of $20 \mathrm{~kg}(200 \mathrm{~N})$ at a crosshead speed of $1 \mathrm{~mm} / \mathrm{min}$. The test platform was changed from the ISO 4049 standard and was based on the platform used by Braz et al. (7). 
It was made of machined stainless steel and aluminum, and comprised a knife that conveyed the tension to the specimen and a base with 2 supports, in which the distance from center to center was $20 \mathrm{~mm}$ (Fig. 1).

From the obtained data at the moment of fracture through the 3 point bending tests, the mechanical cycling for samples with fiber reinforced was conducted in the same test machine, with the same load cell and velocity but using $60 \%$ of maximum tension obtained in the 3 point bending tests for promote crack propagation without fracture. As the objective of the study was not specifically to evaluate the mechanical strength, then these tests were carried out based on the parameters obtained from the 3-point bending tests using samples without fiber reinforcement. After these initial parameters, samples with fiber-reinforcement were submitted to a regimen of up to 200 cycles, and evaluated by OCT, SEM and OM.

\section{OCT Analysis}

Images were obtained of each sample in two moments: before and after the mechanical cycling. Scanning images were obtained sequentially, from left to right, at $250 \mu \mathrm{m}$ intervals between the sectional images.

The experimental OCT setup was similar to the one used before (7) in the spectral domain (SD-OCT). The SD-OCT setup (Fig. 2) uses a broadband light source (Superluminescent Diode; Broadband SLD Lightsource S840; Superlum, Moscow, Russia) operating at 840

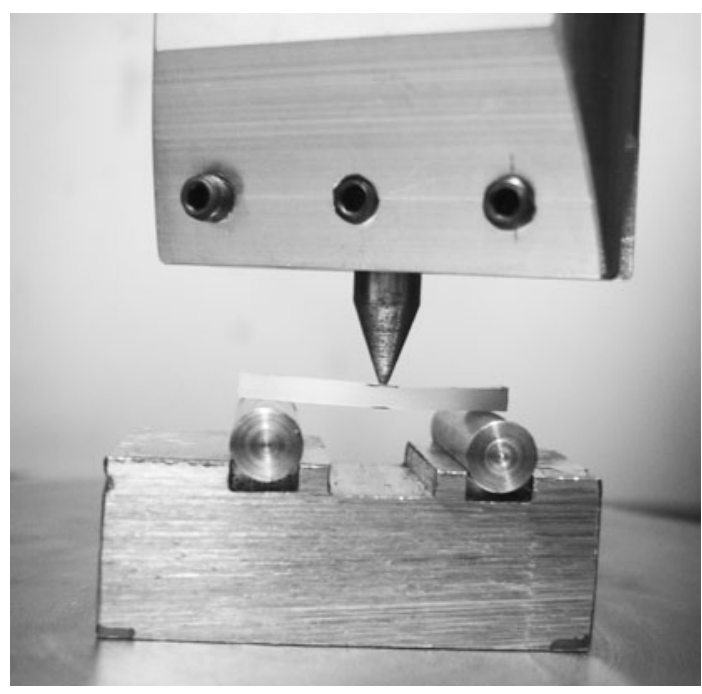

Figure 1. Test platform. $\mathrm{nm}$, spectral width of $50 \mathrm{~nm}$, fiber output power of 25 $\mathrm{mW}$ and a measured spatial resolution of $10 \mu \mathrm{m}$. The system is based on the Michelson interferometer setup: in the reference arm, there is a mirror mounted on a piezoelectric base; in the sample arm, a mirror controlled by a Galvo motor is required for the scanning of light at the sample. Light from the source is first split by a beam splitter and travels down the reference and sample arms. The reflected and backscattered light coming from both arms are then recombined at the beam splitter and collected by a spectrometer, consisting of a lens collimator system, a diffraction grating $(1200 \mathrm{~L} / \mathrm{mm})$, and an optical detector (linear CCD cam, ATMEL, 2048 pixels, 12 bits; Atmel, San Jose, CA, USA), connected to a computer. The system is controlled by the software OCT 800 - Complex Square, developed from LabView program language, which collects data and generates the image. The image acquisition time is less than $1 \mathrm{~s}$.

\section{SEM and OM Analyses}

After evaluation by OCT, specimens whose surface was more homogeneous were selected to analysis by SEM, at the Materials Science and Microscopy Laboratory of CETENE (Centro de Tecnologias Estratégicas do Nordeste). Fragments of about $10 \mathrm{~mm}$ in length were cut for manual polishing with diamond paste $1 \mu \mathrm{m}$ and distilled water, followed by colloidal silicon with particle size about $50 \mathrm{~nm}$. Samples were washed in ultrasonic bath before being taken under the microscope. There was no need to metallization of the samples because the electron microscope employed allows the analysis in low vacuum. For OM, the samples

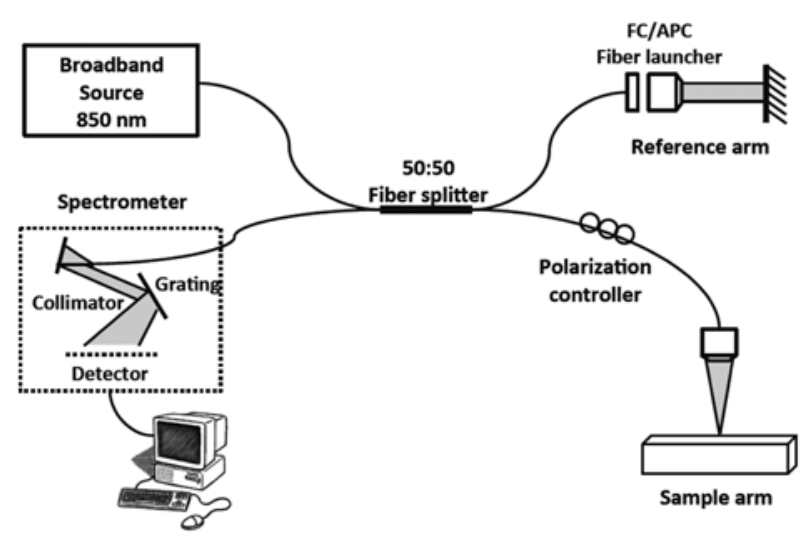

Figure 2. Schematic setup of SD-OCT $840 \mathrm{~nm}$. 
were washed only by ultrasonic bath and were stored in clean and dry ambient.

\section{RESULTS}

Results of the 3-point bending test are shown in Table 1.

The samples submitted to bending tests were made without glass fiber, and those submitted to mechanical cycling had a pre-impregnated fiber internally. It was possible for some specimens to visualize the crack propagation macroscopically during the tests and

Table 1. Maximum tension (in MPa) and maximum force (in N) after 3-point bending test and mechanical cycling test.

\begin{tabular}{ccccc}
\hline & Max. & Max. & & \\
& tension \\
$100 \%$ & tension & & Max. force \\
& $60 \%$ & $100 \%$ & Max. force \\
& & & & $60 \%$ \\
\hline $\mathrm{Z} 250$ & 253.7 & 152.2 & 67.7 & 40.5 \\
$\mathrm{Z} 350$ & 319.3 & 191.6 & 85.1 & 50.9 \\
\hline
\end{tabular}
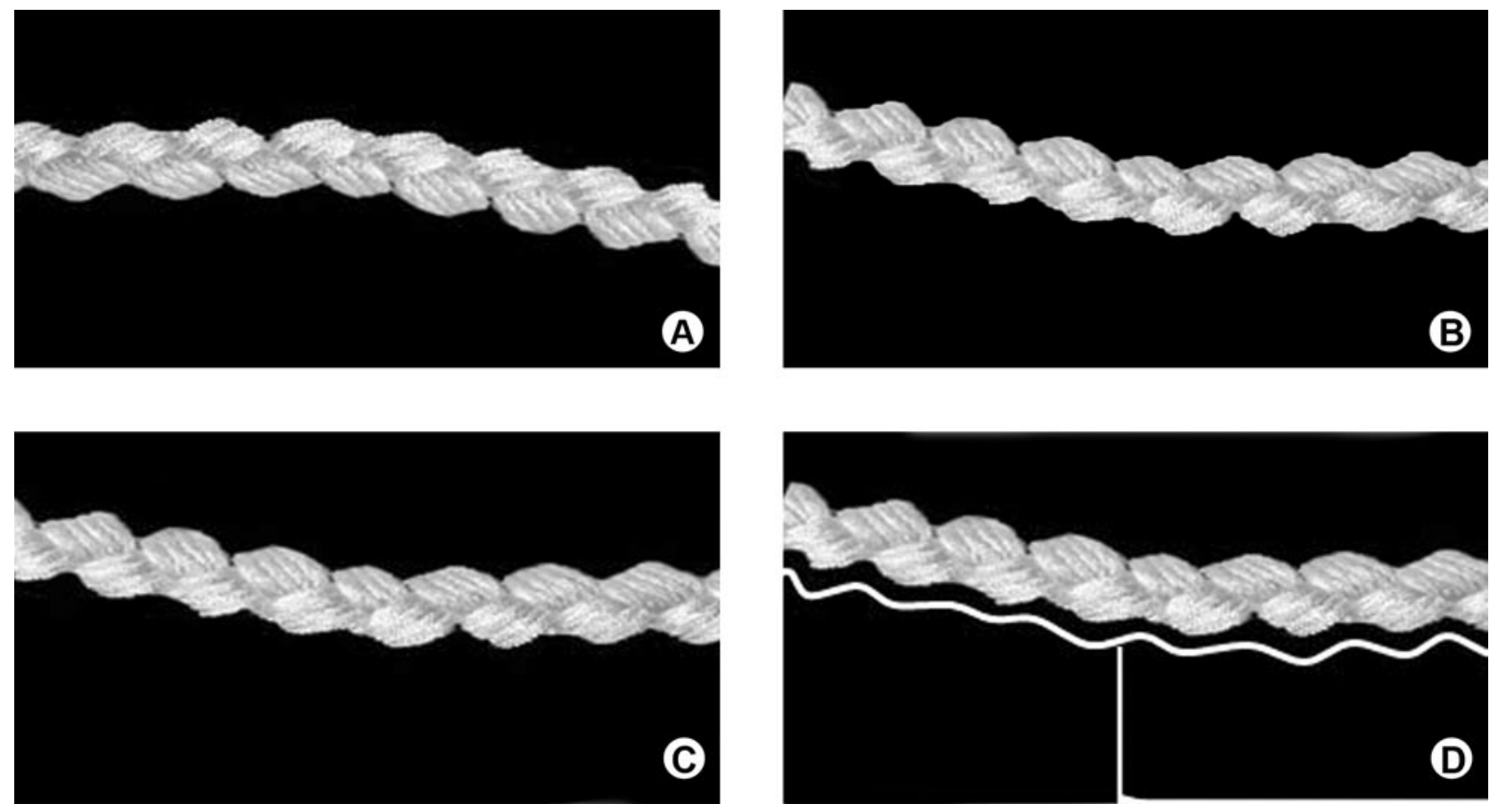

Figure 3. Diagram of the imaging process before cycling test with the fiber inserted (A); during cycling test (B); with the crack propagation (C); and with the extension of crack on the horizontal and vertical directions showing the whole fracture process (D). that case the tests were finished with less than 100 cycles. The samples in which the failure had not begun macroscopically were submitted to 200 cycles also under $60 \%$ of the maximum tension bending tests. After the end of tests, samples were taken for analysis of the fracture process by OCT, SEM and OM. Images before and after mechanical cycling were performed for comparison and to observe the formation of failure process. These images are of the bottom portion of the sample, in other words, the face that develops the tension test.

A diagram was constructed to illustrate how the mechanical cycling test occurs and the process of crack formation and propagation (Fig. 3). In this diagram, it is indicated the deformation occurred in the dental composite and fiber in the direction of the force. The failure may begin at the interface fiber-composite and spread toward the lower surface of the sample, or may originate in the bottom portion and going in the force opposite direction. In the last situation, crack hits the fiber and surrounds the fiber-composite interface.

Figures 4, 5 and 6 present representative examples of OCT images. Figure 4A shows in the internal region the glass fiber found with the braided aspect and a darker color representing the fiber structure. In Figure 4B, 
after mechanical cycling, it is possible to see the same structure without crack propagation, the fiber structure remained without deformation. From bottom to top, in all OCT images, the black region corresponds to the air, the strong white line corresponds to the air-composite interface and the scattering due to the porous composite is seen as the whitish uniform small spots.

Figure 5A represents the image of the bottom portion of the $\mathrm{Z} 350$ composite sample before mechanical cycling. These samples had their test interrupted with 60 cycles because crack propagation was macroscopically observed. The maximum force in which the test has been realized was $50.9 \mathrm{~N}$. It is possible to verify a suggestive image of glass fiber in the internal region, as a darker color indicated by arrow. After mechanical cycling, images were performed in the same region of the sample and it was detected some structures that seems regions of small microcracks, which probably obliterated the transmission of the light producing these apical shadows under them, indicated by the circles (Fig. $5 \mathrm{~B})$. At the bottom portion and on the right hand side of the image, it can be seen a surface defect, probably formed during the production of the sample. In this image, crack propagation in the horizontal direction can be seen surrounding the glass fiber and, in the vertical direction, it appears from the air-composite line perpendicular to the long axis of the sample to the glass fiber. The crack can be identified on the bottom surface through the discontinuity of surface (air-composite line). Using the software Image $\mathrm{J}$ (version 1.32j) the distance
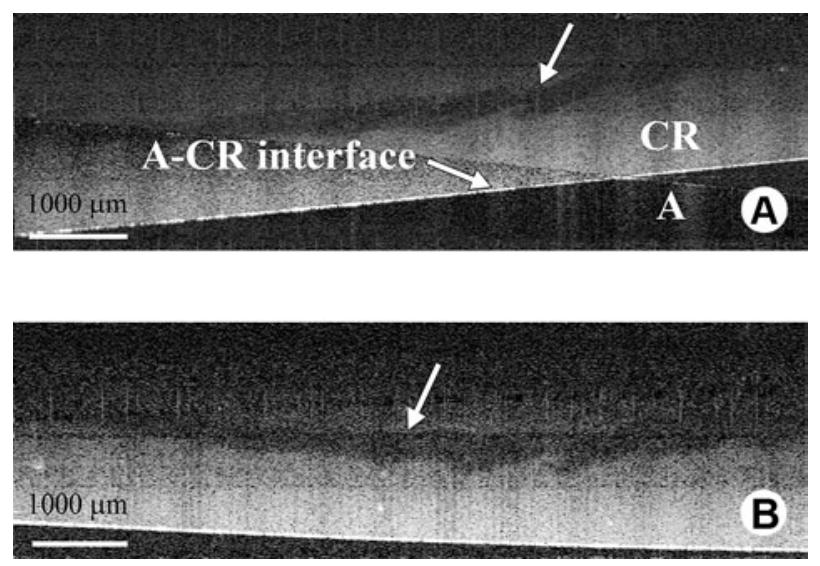

Figure 4. Z250 sample (OCT images). The arrows indicates the region of the fiber insertion featured as a black shadow. $A=$ sample before mechanical cycling test (A: air; A-CR: air-composite resin; $\mathrm{CR}$ : composite resin); $\mathrm{B}=$ after test without crack propagation. between the edges of the crack was measured to be 35 $\mu \mathrm{m}$. The appearance seen in the image in relation to the horizontal crack has an ondulatory form since the fiber has the braided shape, and the distance between the air-composite interface and the glass fiber structure is the $76 \mu \mathrm{m}$.

The bottom portion of the Z350 sample was sanded in order to verify more accurately the horizontal crack propagation. Horizontal crack became more evident because the wavy aspect represents the surface of the glass fiber, localized perpendicular to vertical crack (Fig. 6).

Some samples were imaged under SEM and $\mathrm{OM}$ to analyze the external propagation of cracks. OM images of Z350 sample in Figure 7 show the minimum individual width value at $28.27 \mu \mathrm{m}$ and the maximum at $48.18 \mu \mathrm{m}$. The SEM measurements corresponded to the values obtained by optical microscope: to Z350 sample, the minimal individual value for the width was $35.57 \mu \mathrm{m}$ and the maximum, $43.15 \mu \mathrm{m}$. This means that the superficial failure found in the Z350 sample has the same measurement in both techniques, optical microscopy and SEM.

Thus, the efficiency of the OCT technique on characterization of the dental composite fracture process was confirmed. The measured values were very close when compared with optical and scanning electron microscopy. Using this technique, the distance between the edges of the failure for the Z350 sample was to $35 \mu \mathrm{m}$.
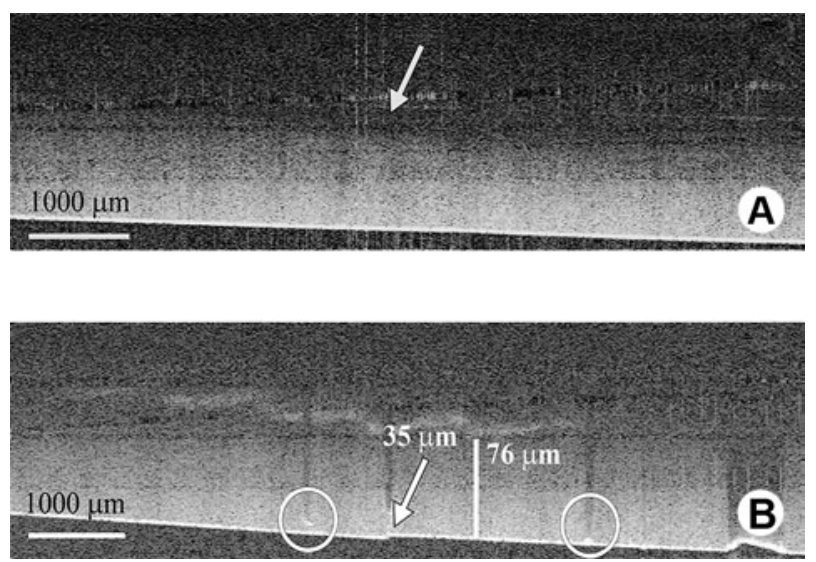

Figure 5. Z350 sample (OCT images). A = before mechanical cycling test; $\mathrm{B}=$ after test showing vertical and horizontal crack propagation. Circles show regions of small microcracks, which probably obliterated the light passage producing these shadows. 


\section{DISCUSSION}

In this work, crack propagation was analyzed qualitatively. The total number of cycles to failure was not the subject of the study. Fatigue research for dental composites usually studies the number of cycles until the occurrence of catastrophic failure (12). The OCT method allows the researcher to follow the fracture process and stop it before the final fracture. Crack initiation is a phase in the fatigue-life of crack that is considered more difficult to predict, since it can depend on microstructural properties. Furthermore, the material is fragile and characterized by catastrophic fracture. The length of the actual initiation period is strongly affected by local microstructural features that may cause localized stress concentrations, such as surface scratches, microcracks, etc (7). This study showed by OCT the fracture process, therefore, this technique can be a very important tool for

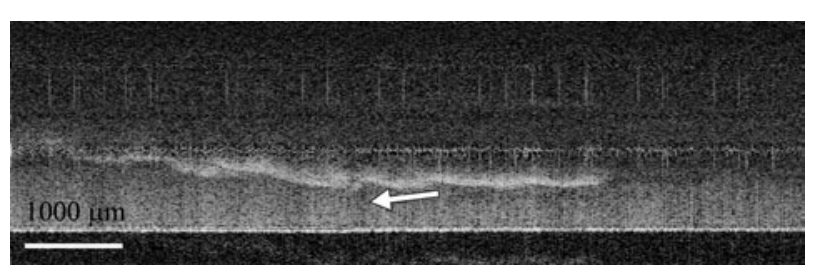

Figure 6. Z350 sample (OCT image) after sanding, showing the crack propagation on the horizontal direction and vertical direction, as indicated by the arrow. failure analysis of materials at the laboratory or at dental clinics, to identify the initial failure of the restorative material before to reach the final fracture, for example.

The analysis of crack propagation using fractographic principles is a well-established analytic tool to determine the failure behavior of brittle materials. According to Le May and Bagnall (13), investigations of structural failure by brittle fracture should take into account, at least, two separate aspects: the first one, the point from which the fracture developed, is relevant in order to determine whether the fracture initiated from a manufacturing effect, whether it experienced a prior fatigue or stress corrosion that originated the crack that led to failure; the second one, it should indicate that, for whatever the defect present, the load applied was enough for unstable fracture to occur.

Brittle materials, such as composites, typically present a population of flaws of different sizes, geometries and orientations. Fracture occurs when the load exceeds a critical value for the propagation of the largest and most favorably oriented flaw (14). These flaws are induced by intrinsic imperfections in the structure of the material, by processing or by mechanical grinding and polishing, and might potentially reduce the strength of the material (15). At composite materials, cracks can originate from the addition particles, for example, inorganic fillers or, in case of metal materials, from grain boundaries and unrecrystallized anisotropic grains (16). In this study, the addition of the glass

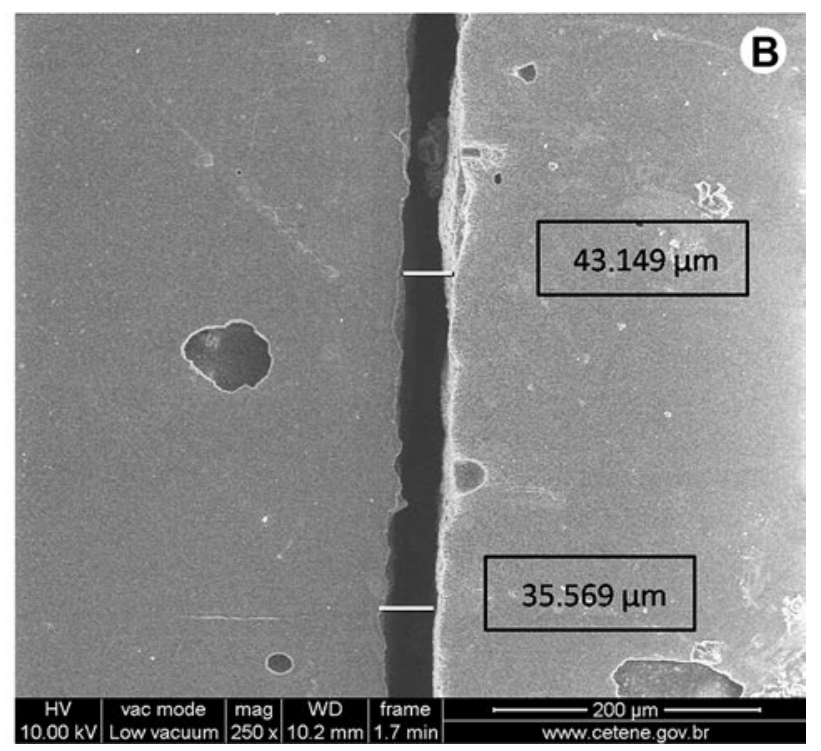

Figure 7. Distance between the edges of the failure in a Z350 sample, as seen by OM (A) and SEM (B). 
fiber into the sample can also act as a point of stress concentration, if there is any failure to filling the matrix with the composite and the fiber, possibly indicating the delamination fracture process, which is a complex fracture mechanism with the formation of transverse cracks initially on the order of the glass fiber (17). This may explain the observation of fracture process in a sample subjected to a low number of cycles.

The light curing process is influenced by the light intensity, time, and output power density used, which consequently influence the mechanical properties of resin materials. A compressive study was performed by Dias and Silva (18), the authors found that the composite resin light-cured with either a halogen lamp or a LED source was similar. However, it may be speculated that the high radiation emitted by the LED in short exposure duration, accelerates the curing reaction, reducing the polymer flowability, increasing the modulus of elasticity and shortening the pre-gel phase, which could interfere with the results of resistance to compression. In our study, the exposure time was increased to $20 \mathrm{~s}$, twice of time. In order to obtain an effective light curing, which means 50 to $60 \%$ of monomer conversion, a radiant energy of approximately $16 \mathrm{~J} / \mathrm{cm}^{2}$ for a 2 -mm-thick resin layer is needed.

With respect to the 3-point bending test, the top surface of the sample is submitted to a state of compression while the bottom surface is submitted to tension test. The stress of maximum tension occurs at the bottom surface of the sample, directly below the application point of force. Limits of tension strength of ceramic materials are equivalent to approximately one-tenth of its compressive strength, so the fracture occurs on the surface of the specimen being submitted to tension (19). By the OCT, SEM and OM analyses, it was possible to verify the outside of cracks on the bottom surface of the sample, where the tension is applied during the mechanical cycling test. However, only through OCT it was possible to examine the material inside and view the extension of the cracks in both horizontal and vertical directions, without damaging the sample. Microscopic analyses allow a more accurate analysis of the sample surface as well as the fracture surface, which is not possible by OCT. The microstructure of materials can also be analyzed microscopically.

The images saved in the OCT's LabView software, a graphical programming development environment for data acquisition, analysis and presentation, has better quality and resolution compared to other programs used for the same purposes. However, it generates too large files. In this study, the images do not have the same high resolution because they had to be converted into files with smaller size using Image $J$ image analysis and processing software for publication purposes. Therefore, it is difficult to characterize some structures that were seen before in images stored in the LabView software. OCT images are very similar to the ultrasound images, for example. Sometimes, some structures can present a more difficult visualization. If we use a laser with greater wavelength, these images can also have a higher resolution. Results obtained indicate that OCT is potentially a powerful technique for visualizing the fracture process. In the same way as OM, OCT provides a laboratory analysis without difficulty, it is easy to use, the samples are easily prepared, and cheaper when compared to SEM. The great advantage of OCT is the three-dimensional image acquisition of internal structures of materials or tissues, without damaging them. Other studies should be performed with same materials for in situ mechanical cycling with the aim of analyzing crack propagation from the start to end.

In this work, three techniques were used to analyze crack propagation in composite resins. SEM and $\mathrm{OM}$ are well established methods for such studies, and we to OCT. In the latter, imaging internally the sample with $10 \mu \mathrm{m}$ axial resolution and penetration depth of $\sim 1.3 \mathrm{~mm}$ presented advantages over the other techniques, which provide mainly images of the surface of the materials. The fractures induced in the samples were observed with all techniques and presented similar results. OCT has also the advantages of being easier to implement and not require any specific preparation of specimens. It can also be used in situ, which opens up new perspective for characterization of dental materials.

\section{ACKNOWLEDGMENTS}

The authors are thankful to CNPq (grant \#35.0313/2008-3) and FACEPE (grant \#APQ-0860-4.02/08), Brazilian Agencies, for financial support. CETENE (Centro de Tecnologias Estratégicas do Nordeste), Physics Department and Mechanical Engineering Department of the Federal University of Pernambuco are gratefully acknowledged, especially Professor Armando Shinohara.

\section{REFERENCES}

1. Anusavice KJ. Phillip's Science of dental materials. $10^{\text {th }}$ ed. Philadelphia, W.B. Saunders Company, 1996.

2. Takeshige F, Kawakami Y, Hayashi M, Ebisu S. Fatigue behavior of resin composite in aqueous environments. Dent Mater 
2007;23:893-899.

3. Baran GR, Boberick KG, McCool JI. Fatigue of restorative Materials. Crit Rev Oral Biol Med 2001;12:350-360.

4. Isaac D. Engineering aspects of the structure and properties of polymer-fibre composites. In: Proceedings of the first symposium on fiber reinforced plastic in dentistry, 1998:1-21.

5. Matheus TCU, Lopes HP, Albuquerque DS, Elias CN, Carmo AMR, Otubo J, et al.. The fracture evaluation of NiTi SMA Endodontics files. Mat Res 2007;10:395-398.

6. Fujimoto JG. Optical coherence tomography for ultrahigh resolution in vivo imaging. Nat Biotechnol 2003;21:1361-1367.

7. Braz AKS, Kyotoku BBC, Braz R, Gomes ASL. Evaluation of crack propagation in dental composites by optical coherence tomography. Dent Mater 2009;25:74-79.

8. Jiao S, Wu C, Knighton RW, Gregori G, Puliafito CA. Registration of high-density cross sectional images to the fundus image in spectral-domain ophthalmic optical coherence tomography. Opt Express 2006;14:3368-3376.

9. Gamelin J, Yang Y, Biswal N, Chen Y, Yan S, Zhang X, et al.. A prototype hybrid intraoperative probe for ovarian cancer detection. Opt Express 2009;17:7245-7258.

10. Hori Y, Yasuno Y, Sakai S, Matsumoto M, Sugawara T, Madjarova VD, et al.. Automatic characterization and segmentation of human skin using three-dimensional optical coherence tomography. Opt Express 2006;14:1862-1877.

11. Melo LSA, Araujo RE, Freitas AZ, Zezell D, Vieira Jr ND, Girkin $\mathrm{J}$, et al.. Evaluation of enamel dental restoration interface by optical coherence tomography. J Biomed Optic 2005;10:064027$1 / 5$.

12. Lohbauer U, Von der Horst T, Frankenberger R, Kramer N, Petschelt A. Flexural fatigue behavior of resin composite dental restoratives. Dent Mater 2003;19:435-440.

13. Le May I, Bagnall C. Brittle fracture and fractography. In: Microscopy, fractography and failure analysis. Boston: International Metallographic Society; 1986. p. IV3-IV24.

14. Ritter JE. Predicting lifetimes of materials and material structures. Dent Mater 1995;11:142-146.

15. Della Bona A, Anusavice KJ, DeHoff PH. Weibull analysis and flexural strength of hot-pressed core and veneered ceramic structures. Dent Mater 2003;19:662-669.

16. Rao KTV, Yu W, Ritchie RO. Cryogenic toughness of commercial aluminum - lithium alloys - role of delamination toughening. Metal Trans A 1989;20:485-497.

17. Kalyanam S, Beaudoin AJ, Dodds Jr RH, Barlat F. Delamination cracking in advanced aluminum-lithium alloys-experimental and computational studies. Eng Fract Mech 2009;2174-2191.

18. Silva CM, Dias KRHC. Compressive strength of esthetic restorative materials polymerized with quartz-tungsten-halogen light and blue LED. Braz Dent J 2009;20:54-57.

19. Callister WD. Fundamentos da ciência e engenharia de materiais, uma abordagem integrada. Rio de janeiro: LTC. 2006.

Accepted September 9, 2010 